\title{
Institutionelle Anbieterkontexte und Regulation von Beratungen in der Weiterbildung
}

\author{
Tim Stanik
}

Eingegangen: 9. März 2015 / Angenommen: 10. Juli 2015 / Online publiziert: 14. August 2015

(C) Die Autor(en) 2015. Dieser Artikel ist auf Springerlink.com mit Open Access verfügbar.

Zusammenfassung Der Beitrag beschäftigt sich mit Beratungen in der Weiterbildung als institutionelle Interaktionsform. In einer vergleichenden Fallstudie wurden verschiedene Daten erhoben, um zu untersuchen, inwiefern die institutionellen Kontexte die Beratungen beeinflussen. Auf der einen Seite definieren die Beratungsanbieter die Beratungsgegenstände und wählen entsprechende Beratende aus. Außerdem verfolgen sie institutionelle Zielsetzungen mit den Gesprächen. Auf der anderen Seite haben die Ratsuchenden eigene Beratungsanliegen und -ziele, mit denen die Beratenden umgehen müssen. Auch wird gezeigt, dass der Beratungsort (ein Anbieter hat seinen Sitz in einem Jobcenter) beeinflusst, wie die Beteiligten miteinander interagieren. Die Ergebnisse machen deutlich, dass sich Beratungen in einem Spannungsverhältnis von institutionellen Kontexten, Erwartungen der Ratsuchenden und dem professionellen Selbstverständnis der Beratenden bewegen.

Schlüsselwörter Weiterbildungsberatung · Bildungsberatung · Institutionelle Interaktion · Forschung

\section{Institutional contexts and regulation of guidance in adult education}

Abstract The article focuses on guidance in adult education as a type of institutional talk. Multiple data sources were collected through a comparative case study of two providers to examine how the institutional contexts influenced the guidance. On the one hand the providers define the subjects of their guidance service and their selection of consultants is based on the content knowledge. Also the providers pur-

T. Stanik $(\bowtie)$

Eberhard Karls Universität Tübingen,

Münzgasse 11, 72070 Tübingen, Deutschland

E-Mail: tim.stanik@uni-tuebingen.de 
sue institutional goals with their guidances. On the other hand the guidance-seekers have their own counselling concerns and goals, which the consultants have to deal with. It is also noted that the location of the guidance (one provider is located in the public employment office) influences how the participants interact. The research results show guidance in adult education is situated in a dialectic relation between the institutional context, the expectations of guidance-seekers and the professional selfconception of consultants.

Keywords guidance $\cdot$ counselling $\cdot$ institutional interaction $\cdot$ adult education research

\section{Einleitung}

Beratungen in der Weiterbildung finden vor dem Hintergrund gesellschaftlicher und institutioneller Anbieterkontexte statt (vgl. Schiersmann 2009, S. 752 ff.). Während die gesellschaftlichen Kontexte einen unmittelbaren Einfluss auf die Beratungsanbieter (z. B. über deren Finanzierung) und somit auch einen mittelbaren auf die Beratungen ausüben, können die Interaktionen von den Beratungsanbietern direkt beeinflusst sein, indem diese nur bestimmte Beratungsformate anbieten oder potenzielle Beratungsgegenstände im Vorfeld festlegen. Durch zeitliche Vorgaben und die Auswahl der Beratenden nehmen die Einrichtungen auch indirekt Einfluss darauf, in welcher Tiefe die jeweiligen Beratungsgegenstände bearbeitet werden können. Außerdem kann sich die jeweilige Kultur der Einrichtung darauf auswirken, wie Beratende die Ratsuchenden wahrnehmen - ob sie beispielsweise eher vorsichtig agieren oder Probleme offen ansprechen (vgl. McLeod und Machin 1998, S. 326 ff.). Die Beratungsanbieter sind aber auch konstitutive und ermöglichende Bedingung dafür, dass ratsuchende Klienten und professionelle Beratende überhaupt zusammenkommen, da sie für die Beratungsangebote Öffentlichkeitsarbeit betreiben, Beratende bestellen und Beratungsräume vorhalten.

Dass mittlerweile ein gesteigertes Bewusstsein über die Bedeutung der gesellschaftlichen und institutionellen Rahmenbedingungen für die Gestaltung von Beratungsprozessen existiert, zeigt sich u. a. in dem vom Nationalen Forum Beratung in Bildung, Beruf und Beschäftigung entwickelten „Kompetenzprofil für Beratende“. Neben Kompetenzen für die Gestaltung der Beratungsprozesse werden sowohl gesellschafts- als auch organisationsbezogene Kompetenzen definiert, um z. B. institutionelle Rahmenbedingungen mitzugestalten, weiterzuentwickeln und ggf. zu verändern (vgl. Haas et al. 2012, S. 18).

Während einige Studien vorliegen, die mikroanalytisch Prozessverläufe von Beratungen in der Weiterbildung untersucht haben (vgl. Disse 2005; Müller 2005; Pörtner 2006; Maier-Gutheil 2009), sind die ganz konkreten Einflüsse der Beratungsanbieter auf die Beratungsprozesse noch nicht empirisch rekonstruiert worden. Betrachtet man aber die bereits vorliegenden Studien unter eben diesem Fokus, wird deutlich, 
- dass sich Beratende an institutionell vordefinierten Beratungsabläufen orientieren (vgl. Maier-Gutheil 2009),

- dass mit Beratungen auch institutionelle Interessen verfolgt werden, die im Widerspruch zu den Erwartungen der Ratsuchenden stehen können (vgl. Disse 2005) oder

- dass die Beratungen von institutionellen Auftraggebern beeinflusst sein können ${ }^{1}$ (vgl. ebd.).

Solche im- und expliziten Einflussnahmen machen es erforderlich, die Wechselwirkungen zwischen den Beratungsanbietern und den darin eingebetteten Beratungsprozessen (vgl. Schiersmann 2011, S. 437 f.) ,kontextsensibel“ zu analysieren (vgl. Käpplinger 2010, S. 33). In diesem Zusammenhang stellt sich dann auch die Frage, wie Beratende vor dem Hintergrund ihres Wissens, ihres Beratungsverständnisses, dem jeweiligen institutionellen Anbieterkontext und den Beratungsanliegen ihrer Klienten professionell agieren.

\section{Anbieter von Beratung in der Weiterbildung}

Beratungen in der Weiterbildung bieten in Deutschland kommunale Weiterbildungsberatungsstellen, Kammern, Arbeitsagenturen, Personal(entwicklungs)abteilungen sowie Weiterbildungseinrichtungen an (vgl. Schiersmann und Remmele 2004, S. 15). Es hat sich bei diesem institutionellen Gefüge etabliert, grundlegend zwischen trägergebundenen und -übergreifenden Beratungen zu unterscheiden. Unter die trägergebundenen werden Beratungen von Weiterbildungseinrichtungen gefasst, die eine „Gelenkstellfunktion“ (Mader 1999, S. 318) haben, indem sie zwischen den Weiterbildungsangeboten der Einrichtungen und der Nachfrage der potenziellen Teilnehmenden vermitteln. Diese Beratungen sind Serviceleistungen für Adressaten und können darüber hinaus einen betriebswirtschaftlichen Nutzen für die Institutionen haben. Als Teil der Distributionspolitik (vgl. Möller 2011, S. 100) ergänzen sie die Öffentlichkeitsarbeit und können zu einer aktiven Bedarfserweckung bei den Ratsuchenden führen (vgl. Faulstich und Zeuner 2010, S. 165).

Unter trägerübergreifende werden dagegen Beratungen von Anbietern gefasst, die nicht selbst Weiterbildungen durchführen, sondern Orientierungs- und Entscheidungshilfen in Weiterbildungsfragen geben. Obwohl die Kammern und die Arbeitsagenturen auch trägerübergreifend beraten, werden insbesondere die kommunalen Weiterbildungsberatungsstellen als Prototyp dieser Beratungen betrachtet. Bei diesen Einrichtungen handelt es sich entweder um eigens für Weiterbildungsfragen geschaffene Beratungsstellen und/oder um Institutionen, die in Netzwerke der regionalen Weiterbildungslandschaft eingebettet sind (vgl. Schiersmann und Remmele 2004, S. 43). Durch trägerübergreifende Beratung soll Entscheidungskompetenz in Weiter-

\footnotetext{
${ }^{1}$ Bei dem untersuchten Gespräch handelt es sich ein Abschlussgespräch einer vom Arbeitsamt geförderten „unspezifischen Feststellungsmaßnahme“. Obwohl es sich um eine Beratung handeln soll, verbindet der Berater mit dem Gespräch die zum damaligen Zeitpunkt vorgeschriebene administrative Aufgabe, einen Eingliederungsplan für die Ratsuchende zu erstellen.
} 
bildungsfragen verbessert, die Bereitschaft zum Lebenslangen Lernens erhöht oder die Nutzung neuer Formen selbstgesteuerten Lernens unterstützt werden (vgl. Deutscher Städtetag o. J.).

Die Differenzierung zwischen trägergebunden und trägerübergreifend suggeriert, dass nur die trägergebundenen Beratungsanbieter wirtschaftlich und rechtlich abhängig von ihrem jeweiligen Träger sind und seine Interessen verfolgen. Im Umkehrschluss würde dies bedeuten, dass trägerübergreifende Beratungsanbieter weder finanziell auf einen Träger angewiesen wären, noch dessen Zielsetzungen berücksichtigen müssten. Die Frage, wo die vermeintlich von Trägern unabhängigen Weiterbildungsberatungsstellen lokal und institutionell angegliedert sind, kann aber durchaus die Gespräche beeinflussen:

Ist eine Beratungsstelle (...) beispielsweise in einem Amt für lokale Arbeitsmarktpolitik angesiedelt, stehen Beratungsbedarfe von Arbeitslosen und Arbeitssuchenden im Vordergrund. Ist eine Weiterbildungsberatungsstelle hingegen etwa in einem Schulamt angesiedelt, spielt (...) der Übergang von Schule bzw. Hochschule und Beruf bei der Beratungsarbeit eine besondere Rolle (Schiersmann und Remmele 2004, S. 43).

Was mit der Unterscheidung trägergebunden vs. -übergreifend zum Ausdruck gebracht werden soll, ist in erster Linie eine Neutralität im Hinblick auf Weiterbildungsmaßnahmen von verschiedenen Weiterbildungseinrichtungen. Daher wird im vorliegenden Beitrag zwischen ,einrichtungsübergreifenden“ und „einrichtungsgebundenen“"Weiterbildungsberatungen differenziert und es wird u. a. dargelegt, inwiefern auch Träger einrichtungsübergreifende Beratungen beeinflussen können. Es werden exemplarisch Beratungen einer kommunalen Weiterbildungsberatungsstelle (WBS) als Repräsentantin einrichtungsübergreifender und Fremdsprachenkursberatungen einer Volkshochschule (VHS) als Repräsentantin einrichtungsgebundener Beratungen analysiert.

\section{Die untersuchten Beratungsanbieter}

Die untersuchte VHS bietet Beratungen in ihren sechs Fachbereichen an, wobei feste Beratungstermine lediglich für den Bereich „Sprachliche Weiterbildung“ existieren. Diese Kurswahl- bzw. Einstufungsberatungen werden zu festen Terminen im Foyer des Hauptgebäudes durchgeführt. Durch diese Verortung wird ein niederschwelliges Beratungssetting arrangiert, da sich dort auch Personen aufhalten können, um sich für Kurse anzumelden oder mithilfe des Programmhefts oder eines PC-Terminals über das Angebot der VHS zu informieren. Für die Beratungen der anderen fünf Fachbereiche existieren keine festen Beratungszeiten. Ratsuchende können sich entweder persönlich zu den Geschäftszeiten der VHS, telefonisch oder per E-Mail an die zuständigen Fachbereichsleitungen wenden. Außerdem ist die VHS anerkannte Beratungsstelle für den Bildungsscheck des Landes und die Bildungsprämie des Bundes.

Die WBS befindet sich in Trägerschaft des kommunalen Jobcenters und eines Netzwerks von regionalen Bildungseinrichtungen. Ein festangestellter Berater bietet personenbezogene und organisationsbezogene Beratungen sowie Förderberatungen 
zum Bildungsscheck und zur Bildungsprämie an. Außerdem werden Beratungen im Auftrag des kommunalen Jobcenters durchgeführt. Personen, die im Arbeitslosengeld-II-Bezug stehen, können von ihren Fallmanagerinnen und -managern zu einer Beratung bei der WBS verpflichtet werden.

Von den jeweiligen Beratungsangeboten der beiden Institutionen werden mit den Fremdsprachenkursberatungen der VHS und den Beratungen der WBS, die nicht auf Anweisung des Jobcenters erfolgen, zwei Beratungsangebote ausgewählt, die von den Ratsuchenden freiwillig in Anspruch genommen werden. Auch müssen in diesen Beratungen nicht wie z. B. bei den Beratungen im Rahmen des Bildungsschecks oder der Bildungsprämie förderpolitische Vorgaben beachtet werden, die die Beratungen regulieren (vgl. Käpplinger und Klein 2013).

\section{Methodische Vorbemerkungen}

Um (regulative) Wechselwirkungen zwischen Anbieterkontexten und (Beratungs-) Interaktionen zu rekonstruieren, werden die Beratungseinrichtungen als Institutionen betrachtet, die nach bestimmen Regeln des Arbeitsablaufs und der Verteilung von Funktionen auf die Mitarbeitenden gesellschaftlich relevante Aufgaben erfüllen (vgl. Tippelt 2009, S. 454). Von einem interaktionistischen Institutionsbegriff ausgehend werden die Institutionen aber nicht als gegebene Tatsachen oder als Orte betrachtet, sondern als Phänomene, die in den Beratungen wechselseitig konstituiert, aufrechterhalten oder modifiziert werden. Es werden dementsprechend die spezifischen Handlungsspielräume und -beschränkungen der Beratungen als ein institutionelles Interaktionsformat betrachtet (vgl. Nolda 2000, S. 74 f.). Dabei steht man unweigerlich vor der methodologischen und methodischen Herausforderung, festzustellen, inwieweit die institutionellen Anbieterkontexte eine tatsächliche oder nur eine scheinbare - möglicherweise vom Interpreten induzierte - Relevanz für die Beteiligten haben. Die untersuchten Beratungen sind nämlich in eine Vielzahl von unterschiedlichen Kontexten eingebettet (Stadt, Region, Lebensumstände etc.). Außerdem haben die Beteiligten neben ihren institutionellen Rollen, „Beratende und Ratsuchende“, auch andere persönliche Merkmale und gesellschaftliche Stellungen etc. inne, die ebenfalls als Kontexte in einer Beratung relevant werden können. Eine Lösungsmöglichkeit besteht darin, die anbieterinstitutionellen Kontexte als etwas zu behandeln, an dem sich die Beteiligten orientieren können, aber nicht müssen, und als etwas, das erst durch das Handeln gemeinsam konstituiert wird (vgl. Drew und Heritage 1992, S. 21). Es genügt also nicht aufzuzeigen, dass die Institutionen relevante Bezugspunkte für die Beteiligten sind, sondern es muss herausgearbeitet werden, wie sich das Institutionelle in den Gesprächen sukzessive darstellt und entwickelt (vgl. Eberle 1997, S. 264). Mit einer solchen streng rekonstruktiven Position, wie sie insbesondere Vertreter der ethnomethodologischen Konversationsanalyse vertreten, ist jedoch die Annahme verbunden, dass Interaktionsanalysen ohne Berücksichtigung des Vorwissens um den jeweiligen institutionellen Kontext durchgeführt werden können. Dies ist auch solange möglich, wie formale Mechanismen der Gesprächsorganisation rekonstruiert werden. Werden die Erkenntnisinteressen um inhaltliche Fragestellungen erweitert, können diese erst durch die Hinzunahme von weiteren 
Daten beantwortet werden (vgl. Deppermann 2000, S. 105). Eine solche Verknüpfung ist insbesondere auch dann notwendig, wenn, wie im vorliegenden Beitrag, institutionelle Rahmenbedingungen professionellen Handelns untersucht werden (vgl. Deppermann 2010, S. 15). Daher werden gemäß einer ,ethnographischen Gesprächsanalyse“ (vgl. Deppermann 2000) nicht nur Ausschnitte aus Beratungsgesprächen, sondern auch die jeweiligen institutionellen Selbstdarstellungen der Beratungsangebote, eine qualitative Befragung der Beratenden und Informationen über die Beratungssettings datentriangulativ hinzugezogen.

\section{Institutionelle Regulationen}

Im Folgenden wird dargelegt, wie die Anbieter die Beratungen regulieren, indem sie versuchen, die Beratungsgegenstände einzugrenzen und Beratende mit entsprechendem Wissen einzusetzen. Es wird außerdem aufgezeigt, dass mit den Beratungen auch institutionelle Interessen verfolgt werden und es wird dargestellt, inwiefern auch die Träger auf einrichtungsübergreifende Beratungen indirekt Einfluss nehmen können.

\subsection{Institutionelle Eingrenzungen der Beratungsgegenstände}

Sowohl die VHS als auch die WBS stellen sich und ihr Beratungsangebot in Selbstdarstellungen vor. Die VHS wirbt auf einer Seite in ihrem Programmheft für die Fremdsprachenkursberatungen, und die WBS hat einen Einrichtungsflyer veröffentlicht. Neben den Beratungsorten und Beratungszeiten werden in diesen Selbstdarstellungen bereits die adressierten Ratsuchenden und die potenziellen Beratungsgegenstände eingegrenzt. Während sich die Fremdsprachenkursberatungen ausschließlich an Personen mit fremdsprachlichen Vorkenntnissen richten, die einen entsprechenden Kurs belegen wollen, adressiert die WBS gemäß ihres Flyers insbesondere Personen, die Fragen zu beruflichen Weiterbildungen haben und/oder vor einer Weiterbildungsentscheidung stehen. Als Beratungsgegenstände werden demensprechend Fragen zur beruflichen Neu- und Umorientierung, zur Auswahl von Weiterbildungseinrichtungen, zu Weiterbildungsmaßnahmen und deren Finanzierungsmöglichkeiten genannt.

Dass diese institutionellen Eingrenzungen der Beratungsgegenstände auch in der Interaktion relevant werden, zeigt der folgende Ausschnitt einer Fremdsprachenkursberatung.

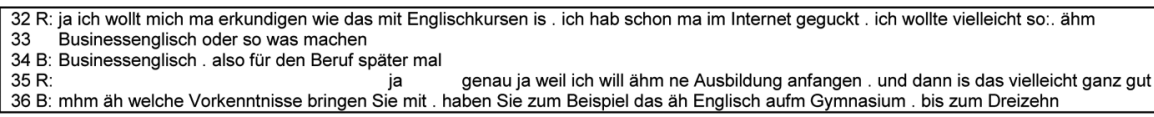

(Fremdsprachenkursberatung 10, VHS)

Die Ratsuchende gibt das Thema der Beratung vor - sie möchte sich über Businessenglischkurse informieren. Nachdem die Beraterin versichernd nachgefragt hat, ob sie Englisch für eine Berufstätigkeit lernen möchte, begründet die Ratsuchende ihren Entschluss mit der Aufnahme einer Berufsausbildung. Offensichtlich weiß sie aber 
noch nicht, welchen Ausbildungsberuf sie anstrebt und ob ihr tatsächlich ein berufsbezogener Englischkurs dabei nützen könnte. Auf dieses potenzielle Beratungsanliegen geht die Beraterin nicht ein, sondern erkundigt sich nur nach den Vorkenntnissen der Ratsuchenden. In der Beratung wird schließlich ein Kurs für die Ratsuchende gefunden, der berufsbezogen ist, ihren Vorkenntnissen entspricht und zu dem sie sich auch anmelden will. Bei der Kursbuchung wird deutlich, dass die Ratsuchende einen Studentenausweis besitzt und offensichtlich ihr Studium zugunsten einer Ausbildung abbrechen will. Das Nicht-Aufgreifen dieser potenziellen Beratungsgegenstände „Aufnahme einer Berufsausbildung“ bzw. „Studienabbruch“ entspricht zum einen einer Kurswahlberatung und zum anderen erhält die Beraterin für deren Bearbeitung keinen expliziten Auftrag. Dass solche potenziellen Beratungsgegenstände jedoch auch nicht von den Beraterinnen bearbeitet werden, wenn diese explizit von den Klienten eingebracht werden, zeigt ein anderer Fall. Bereits zu Beginn dieser Beratung gibt die Ratsuchende an, im Rahmen einer Maßnahme der Agentur für Arbeit aufgefordert worden zu sein, einen abschlussbezogenen Englischkurs zu besuchen, um ihre Beschäftigungschancen zu erhöhen. Sowohl die aktuelle Lebenssituation „Arbeitslosigkeit“ als auch das Motiv „Zertifikatserwerb“ werden von der Beraterin nicht aufgegriffen. Erst als die Ratsuchende die Beraterin wiederholt danach fragt, ob sie eine Englischanfängerkurs-Teilnahmebescheinigung für eine Bewerbung nutzen könne, antwortet diese:

$141 \mathrm{~B}:$ da sind dreißig Unterrichtsstunden nich so beeindruckend für nen Arbeitgeber .. net natürlich

$142 \mathrm{R}: \quad$ ich will da richtig drin bleiben

$143 \mathrm{~B}$ : äh . is es schön wenn man die Teilnahmebescheinigung dann reinlegt . in die Bewerbungsunterlagen, ansonsten muss man aber auch 144 weiter lernen

(Fremdsprachenberatung 1, VHS)

Es zeigt sich, dass die Beraterin durchaus Wissen über den Arbeitsmarkt hat, da sie einschätzt, wie potenzielle Arbeitgeber die Aussagekraft eines Englischanfängerkurses bewerten. Beachtenswert ist, dass sie diese Einschätzung trifft, ohne danach gefragt zu haben, in welchem Berufsfeld die Ratsuchende tätig werden will. So könnte eine Englischanfängerkurs-Teilnahmebescheinigung für die Bewerbung als Außenhandelskauffrau nicht „,beeindruckend“ für einen potenziellen Arbeitgeber sein, während diese bei der Bewerbung um eine Stelle als Lagerarbeiterin eine besondere Qualifikation darstellen könnte. Im Interview gibt die Beraterin auf diesen Fall angesprochen an, keine „Arbeitsberaterin“ zu sein und darum nicht beurteilen zu können, ob englische Sprachkenntnisse Berufschancen tatsächlich erhöhen.

Auch in den Beratungen der WBS werden insbesondere von arbeitslosen Ratsuchenden potenzielle Beratungsgegenstände deutlich, die nicht denen der institutionellen Selbstdarstellung entsprechen. Auf die Frage des Beraters, warum z. B. eine arbeitslose Ratsuchende im ,sozialen Bereich“ tätig werden will, beschreibt diese ihre Biografie: ein Selbstmordversuch der Mutter, Verlust der Kindheit, alkoholkranker Vater und Trennung von ihrem Bruder werden genannt. Der Berater reagiert zurückhaltend mit Hörerrückmeldungen und stellt keine Nachfragen: 


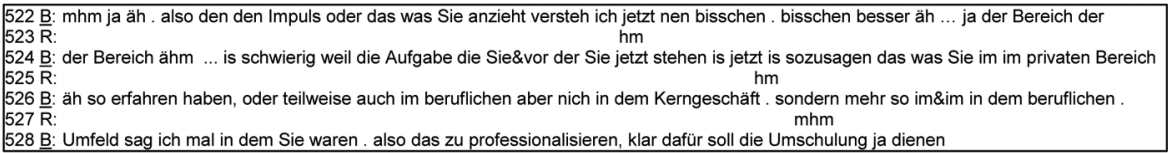

(Beratung 4, WBS)

Die höchst problematischen Schilderungen werden schließlich als „Impuls“ bezeichnet und somit nicht zu Gegenständen der Beratung. Abstrahierend von den biografischen Schilderungen nennt der Berater die Herausforderung, dass sich die Ratsuchende „professionalisieren“ müsse. Dabei erwähnt er die Möglichkeit einer Umschulung und führt das Gespräch zurück auf einen von der Einrichtung festgelegten Beratungsgegenstand. Damit wahrt der Berater aber auch eine professionelle Distanz. So gibt er im Interview an, aus seinem „Laienverständnis“ heraus weder „psychologische Störungen“ zu diagnostizieren noch zu versuchen, den Ratsuchenden bei tiefliegenden Problemen zu helfen.

\subsection{Institutionelle Auswahl der Beratenden}

Die VHS setzt in ihren Fremdsprachenkursberatungen ausschließlich nebenberufliche Fremdsprachenkursleiterinnen ein. Diese Beraterinnen haben Wissen über Fremdsprachenlernen und verfügen über Erfahrungen mit den Ratsuchenden als potenzielle Kursteilnehmende.

Dies ist solange förderlich, wie sich die Fragen der Ratsuchenden ausschließlich auf das Fremdsprachenlernen und eine Kurswahl bei dieser VHS beziehen. Die Ratsuchende des nächsten Ausschnitts erkundigt sich jedoch nach der Möglichkeit, einen von der Beraterin vorgeschlagenen Bildungsurlaub mithilfe des Bildungsschecks des Landes zu finanzieren und bietet damit eine Lösung für ein angedeutetes Finanzierungsproblem an.

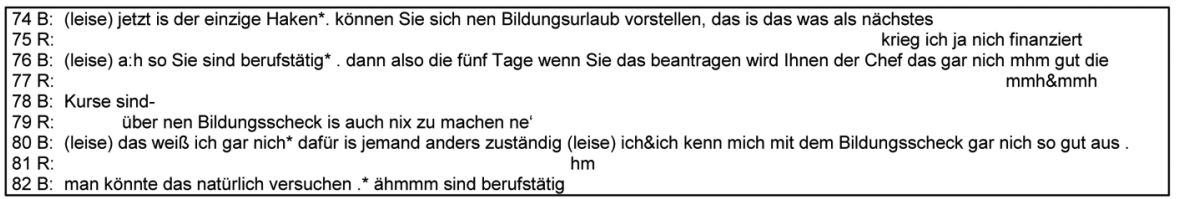

(Fremdsprachenkursberatung 2, VHS)

Die Beraterin kann diese Frage aufgrund der innerinstitutionellen Arbeitsteilung nicht beantworten. Auffällig ist, dass die Beraterin nicht an eine dafür zuständige Stelle delegiert, sondern sich danach erkundigt, ob die Ratsuchende berufstätig sei. Auch im weiteren Verlauf wird der von der Ratsuchenden eingebrachte Beratungsgegenstand „Bildungsscheck“ nicht bearbeitet. Stattdessen wird ein kostengünstiger Kurs gefunden, zu dem sich die Ratsuchende auch schließlich anmeldet. In einem anderen Fall erkundigt sich eine Ratsuchende danach, ob sie einen Englischanfängerkurs mit dem einer anderen Einrichtung kombinieren könne. Sie formuliert damit einen einrichtungsübergreifenden Beratungsauftrag. Auch dieser wird von der Beraterin mit dem Hinweis auf ihr Nichtwissen über den Kurs bei der Konkurrenzeinrichtung abgelehnt: ,weil ich nich weiß, was in dem Institut oder in der Schule bei 
der Sie sich erkundigt haben, durchgenommen wird, wie viele Stunden das sind, mit welchem Buch die arbeiten“ (Fremdsprachenkursberatung 1, VHS, Z. 94 f.). Beachtenswert ist, dass die Beraterin indirekt zu erkennen gibt, dass sie mithilfe dieser Informationen durchaus in der Lage wäre, dieses Beratungsanliegen zu bearbeiten. Erneut darauf im Interview angesprochen, gibt sie an, dass sie es sich mal vorgenommen habe, sich über Angebote anderer Weiterbildungseinrichtungen zu informieren, davon aber Abstand genommen habe, da dies nicht ihre Aufgabe sei.

Der Berater der WBS hat vor seiner Tätigkeit als Weiterbildungsberater bei der Agentur für Arbeit als Projektleiter gearbeitet. Dass das dort erworbene Wissen auf sein Beratungshandeln Einfluss hat, zeigen insbesondere die Beratungen von arbeitslosen Ratsuchenden. Während die Fremdsprachenkursberaterinnen Beratungsanliegen aufgrund ihres Nichtwissens nicht aufgreifen, bringt der Berater in diesen Fällen stellvertretend Beratungsgegenstände ein. So ist eine obligatorische Frage des Beraters, ob und in welchem Kontakt die Ratsuchenden zu ihren Fallmanagern stehen. Einer arbeitslosen Ratsuchenden, die schon seit über einen Jahr keinen Termin mehr bei der Agentur für Arbeit hatte, wird daraufhin empfohlen, den Kontakt zu ihrer Fallmanagerin wieder aufzunehmen, um dieser zu zeigen, dass sie eine Beschäftigung aufnehmen möchte und selbst aktiv wird.

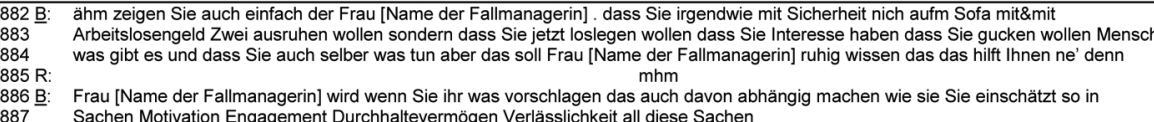

\section{(Beratung 1, WBS)}

Dies wird damit begründet, dass die Fallmanagerin ein Ermessen bei der finanziellen Bewilligung einer Umschulung ausüben könne und dies auch auf einer subjektiven Einschätzung der Ratsuchenden beruhe. Streng genommen hat der Berater dafür weder einen Auftrag der Ratsuchenden noch der Institution. Ebenfalls darauf im Interview angesprochen, gibt er an, mit arbeitslosen Ratsuchenden eine ,Taktikberatung“ durchzuführen, um ihnen zu vermitteln, wie ein „Fallmanager tickt“. In Anlehnung an Becker-Mrotzek (2001, S. 1522) lässt sich dieses Vorgehen als eine „behördliche Beratung im weiteren Sinne“ charakterisieren, da arbeitslosen Ratsuchenden Hilfestellungen im Umgang mit Mitarbeitern des Jobcenters gegeben werden. ${ }^{2}$ Dieses Vorgehen macht der Berater nach eigener Aussage gegenüber dem Jobcenter transparent, indem er an die Fallmanager und -managerinnen appelliert: „Einen kompetenten Kunden habt ihr doch viel lieber als nen dummen Kunden.“

Fragen der Ratsuchenden, die eine konkrete Berufswahl oder Weiterbildungsmaßnahme betreffen, werden zwar vom Berater aufgegriffen, aber modifiziert. So führt er weder eine konkrete Berufs- noch Kurswahlberatung durch, sondern zeigt ihnen exemplarisch, wie sie mithilfe von Internetdatenbanken der Agentur für Arbeit oder

\footnotetext{
${ }^{2}$ Becker-Mrotzek (2001, S. 1522) unterscheidet bei Beratungsgesprächen in Behörden und Ämtern zwischen Beratungen im weiteren und engeren Sinne. Während bei Beratungen i. w. S. keine Probleme des Bürgers, sondern Schwierigkeiten, die aus der Kooperation mit der Behörde entstehen, bearbeitet werden, sind Beratungen i. e. S. Gespräche, in denen lebensweltliche Probleme gelöst werden.
} 
auf Internetpräsenzen von Weiterbildungseinrichtungen des Netzwerks sich selbst informieren können. Mit Fragen zu konkreten Inhalten von Umschulungen oder zu Zugangsvoraussetzungen werden die Ratsuchenden an die Weiterbildungseinrichtungen verwiesen. Dieses Vorgehen wird im Interview als „Lotsenfunktion“ bezeichnet, da es sich um eine „unspezifische Beratungsstelle“ handelt.

\section{3 (Träger-)Institutionelle Interessen}

Die Fremdsprachenkursberatungen dienen laut Programmheft dazu, dass Ratsuchende einen Kurs belegen, der ihren Vorkenntnissen entspricht. Dass damit nicht nur die Ratsuchenden bei einer Kursentscheidung unterstützt werden, sondern auch ein institutionelles Interesse verfolgt wird, zeigt sich daran, dass in den Beratungen ausschließlich Kurse der eigenen Einrichtung vorgeschlagen werden. Die Beraterinnen gehen auch dann so vor, wenn Weiterbildungsinteressen und -ziele der Ratsuchenden nicht mit dem Angebot der VHS übereinstimmen. Die Ratsuchende des folgenden Ausschnitts benötigt beispielsweise für das Nachholen ihrer mittleren Reife die Zertifizierung ihrer Englischkenntnisse auf einer bestimmten Niveaustufe:

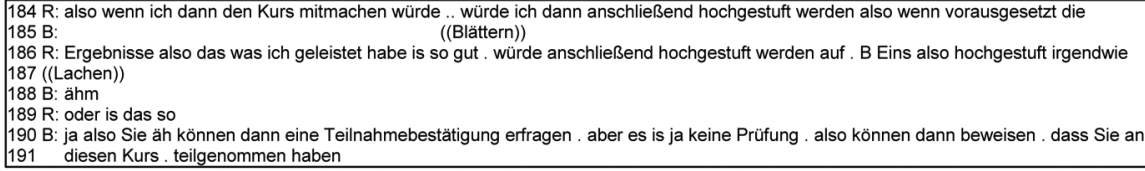

(Fremdsprachenkursberatung 13, VHS)

Erst auf Nachfrage der Ratsuchenden gibt die Beraterin zu, dass die Ratsuchende nach einem Kursbesuch lediglich eine Teilnahmebestätigung erhalten könne und diese auch nur auf ihren ausdrücklichen Wunsch hin ausgestellt werde. Die Beraterin versucht, die Ratsuchende durch das Zurückhalten dieser Information zu einem Kursbesuch bei der VHS zu veranlassen, obwohl sie damit ihr Weiterbildungsziel nicht erreichen kann. Auffällig ist aber, dass sie nicht darauf aufmerksam macht, dass Teilnehmende nach einem Kursbesuch eine separate Prüfung bei der VHS ablegen können. Offensichtlich dienen die Beratungen ausschließlich dazu, eine kurzfristige Kursentscheidung herbeizuführen. Auch wenn Ratsuchende nur ihre Vorkenntnisse eingestuft bekommen möchten, wird mit ihnen nach der Absolvierung des Einstufungstests eine Kurswahlberatung durchgeführt. Mit der Inanspruchnahme einer Fremdsprachenkursberatung werden alle Ratsuchenden zu potenziellen Teilnehmenden, mit denen auch ohne ihren Auftrag eine einrichtungsgebundene Kurswahlberatung durchgeführt wird. Einschränkend muss aber gesagt werden, dass die vorliegenden Beratungsfälle nicht ,missbraucht“ werden, um - wie von Tippelt (1997, S. 14) vermutet - Kurse gezielt auszulasten. Stattdessen werden den Ratsuchenden Kurse aus dem gedruckten Programmheft vorgeschlagen, eine Kursentscheidung wird aber ausdrücklich an sie übertragen. Im Interview gibt eine Beraterin aber auch an, dass sie versucht, z.B. Seniorinnen und Senioren ,diskret in Vormittagskurse reinzuschleusen“, da sie dort besser aufgehoben wären. 
Wie dargelegt, sind die Träger der WBS regionale Bildungseinrichtungen und das kommunale Jobcenter. Während der Berater sich gegenüber den Angeboten der regionalen Bildungseinrichtungen neutral verhält, indem er z. B. den Ratsuchenden empfiehlt, sich bei mehreren Anbietern zu erkundigen, nimmt er in Beratungen von arbeitslosen Ratsuchenden eine vermittelnde Position zwischen ihnen und dem Jobcenter ein. Er versucht, Vorgehensweisen und Entscheidungen des Jobcenters zu rechtfertigen und Vorurteile der Ratsuchenden gegenüber der Behörde abzubauen. Obwohl alle arbeitslosen Ratsuchenden der mitgeschnittenen Beratungen gewillt sind, ihre berufliche Situation zu verändern und sich bereits über Weiterbildungsmaßnahmen informiert haben, ist ein weiteres Ziel, die „Aktivierung und Motivierung von Arbeitslosen“"

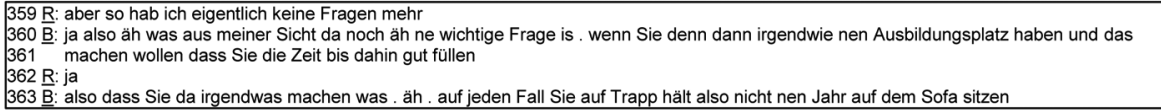

363 B: also dass Sie da irgendwas machen was. äh . auf jeden Fall Sie auf Trapp hält also nicht nen Jahr auf dem Sofa sitzen

(Beratung 2, WBS)

Die Anbahnung des Gesprächsabschlusses durch den Ratsuchenden wird vom Berater in diesem Ausschnitt nicht aufgegriffen, sondern er thematisiert die aus seiner Sicht ,wichtige Frage“, dass der Ratsuchende sich bis zum Ausbildungsbeginn um eine Beschäftigung bemüht. Dieses Vorgehen lässt sich auf den Träger „Jobcenter“" zurückführen, der gemäß seines gesetzlichen Auftrags um die Aktivierung von Arbeitslosen bemüht zu sein hat. Mit den Beratungen werden aber auch Interessen der eigenen Institution verfolgt. So wird allen Ratsuchenden die Möglichkeit eröffnet, eine Folgeberatung in Anspruch zu nehmen. Einerseits zeigt der Berater damit seine Bereitschaft, die Ratsuchenden langfristig zu begleiten und zu unterstützen. Andererseits könnte es sich um ein Vorgehen handeln, um die Beratungsstelle langfristig zu sichern. Die WBS ist laut Angaben des Beraters im Interview als ein ,nicht befristetes Projekt"“ angelegt, was zur Folge habe, dass die Einrichtung leichter wieder abzuschaffen sei. So muss die WBS auch jedes Jahr in einem Geschäftsbericht ausweisen, wie viele Beratungen durchgeführt wurden. Diesem Bericht ist zu entnehmen, dass die WBS mit den Förderberatungen im Jahr der Erhebung knapp $12.000 €$ erwirtschaften konnte. Dies sind auch die einzigen Einnahmen, die nicht von den Trägern stammen. Vor diesem Hintergrund fällt auf, dass der Berater in einigen Fällen darauf aufmerksam macht, dass die WBS auch Förderberatungen anbietet, wobei jedoch ein gesonderter Termin vereinbart werden müsse. Auf die Möglichkeit, sich bei Einrichtungen des Netzwerks beraten zu lassen, wird dagegen nicht hingewiesen.

\section{4 Örtliche Angliederung der WBS an den Träger}

Die Beratungen der WBS werden in einem Büroraum durchgeführt, der sich im Gebäude des kommunalen Jobcenters befindet. Diese örtliche Angliederung beeinflusst den Berater in seinem Beraterverhalten offensichtlich, da er ,Taktikberatungen“ durchführt und zwischen Ratsuchenden und Jobcenter vermittelt. Im Interview gibt er außerdem zu, dass, wenn „Gelder knapp werden und Umschulungen nur noch ganz wenig gemacht werden“, er arbeitslosen Ratsuchenden nicht zu einer Umschu- 
lung raten könne. Dass die örtliche Angliederung ans Jobcenter nicht nur sein Verhalten, sondern auch das der Ratsuchenden beeinflussen kann, zeigt sich insbesondere zu Beginn der Beratungen von arbeitslosen Ratsuchenden. Diese beginnen nämlich auf die immer gleiche, unspezifische Eingangsfrage des Beraters, ,was er für die Ratsuchenden tun könne“, ihre Berufsbiografien zu schildern. Dabei begründen sie narrativ überschüssig ihr aufrichtiges Interesse an der Aufnahme einer Beschäftigung. Einige haben sogar ihre vollständigen Bewerbungsunterlagen dabei. Erst im Vergleich mit den Fremdsprachenkursberatungen zeigt sich daran ein Einfluss des Trägers ,Jobcenter", mit dem die Ratsuchenden wahrscheinlich aufgrund ihrer Arbeitslosigkeit regelmäßig in Kontakt gekommen sind. Weder schildern die Ratsuchenden der Fremdsprachenkursberatungen ihre Berufsbiografien noch bringen sie ihre Zeugnisse mit. Auf die Interviewfrage, ob der Sitz der Weiterbildungsberatungsstelle im Gebäude des Jobcenters problematisch für seine Arbeit sei, antwortet der Berater mit der paradoxen Interjektion ,jein“" und bemerkt, Acht geben zu müssen. Vorsichtig formuliert er, dass „möglicherweise“ eine Angliederung an die städtische Wirtschaftsförderung oder an eine Volkshochschule besser wäre. So ist der Berater in diesen Fällen gefordert, den institutionellen Kontext Weiterbildungsberatungsstelle vom trägerinstitutionellen Kontext Jobcenter abzugrenzen und auf die vertrauliche Behandlung der Gespräche gegenüber den Fallmanagerinnen und -managern hinzuweisen.

\section{Fazit und Forschungsbedarfe}

Die Analysen zeigen, dass sich Beratungen in der Weiterbildung in einem Spannungsverhältnis zwischen den (träger-)institutionellen Kontexten, den Beratungsgegenständen der Ratsuchenden und dem professionellem Handeln der Beratenden bewegen. Die Beratungsanbieter nehmen auf der einen Seite durch eine Eingrenzung der Beratungsgegenstände und durch die Auswahl der Beratenden Einfluss darauf, was überhaupt Gegenstand einer Beratung werden kann. Auf der anderen Seite agieren die Beratenden aber auch professionell, indem sie die Beratungsanliegen ,so zu definieren vermögen, dass diese eben möglichst weitgehend den Lösungen entsprechen, über die sie je (professionell) verfügen“ (Pfadenhauer und Brosziewski 2008, S. 82). In diesem Zusammenhang zeigt sich, dass institutionelle Eingrenzungen von Beratungsgegenständen bei Weiterbildungsfragen insofern schwierig sind, als solche immer auch (berufs-)biografische Probleme oder Ziele implizieren können. Für solche Fälle bedarf es Beratender, die nicht nur eine professionelle Distanz wahren, Beratungsanliegen im Rahmen ihres professionellen Wissens bearbeitbar halten, sondern auch solche, die Ratsuchende an entsprechende Institutionen verweisen können (vgl. Stanik 2014, S. 142). Hierzu ist es notwendig, dass Beratende eingesetzt werden, die nicht nur über das notwendige Fachwissen, sondern auch über hinreichende Beratungskompetenzen verfügen. Da Beratungen in der Weiterbildung bislang nicht marktfähig sind, bedarf es gezielter Förderung von Beratungsangeboten, die von öffentlichen Weiterbildungseinrichtungen durchgeführt werden.

Während es nicht weiter überrascht, dass die VHS die Beratungsgegenstände der Fremdsprachenkursberatungen stark thematisch eingrenzt und nicht einrichtungsübergreifend berät, fällt bei den Beratungen der WBS auf, dass diese auch durch 
ihren Träger, dem kommunalen Jobcenter, beeinflusst werden. Daher lassen sich die Beratungen der untersuchten Weiterbildungsberatungsstelle zwar weiterhin als „einrichtungsübergreifend“, aber eben auch als „trägerorientiert“ charakterisieren. Es bleibt zu prüfen, inwiefern sich eine solche ex- und implizite Trägerorientierung aufseiten der Ratsuchenden in anderen vermeintlich von ihrem Träger unabhängigen Weiterbildungsberatungsstellen identifizieren lassen. Auch wäre zu untersuchen, inwieweit Beratungshandeln aufgrund der Berufsbiografien der Beratenden und/oder der Verortung ihrer Beratungsstellen bei anderen Trägern beeinflusst wird. Die rechtliche und örtliche Angliederung der untersuchten Weiterbildungsberatungsstelle an das kommunale Jobcenter ist nur eine Variante. Andere kommunale Weiterbildungsberatungsstellen sind beim Schulamt, beim Bildungsdezernat oder der Wirtschaftsförderung angesiedelt (vgl. Schiersmann und Remmele 2004, S. 43). Hierzu bedarf es Analysen, die Beratungen von Weiterbildungsberatungsstellen mit unterschiedlicher Trägerschaft vergleichend untersuchen. Auch der Umstand, dass mit den untersuchten Beratungen nicht nur Interessen der Ratsuchenden, sondern auch institutionelle verfolgt werden, könnte Anlass für weitere Untersuchungen sein, die rekonstruieren, inwiefern institutionsbezogene Interessen auch von anderen Beratungsanbietern verfolgt und wie solche Interessen mit den Beratungsanliegen der Ratsuchenden in den Beratungsinteraktionen verschränkt werden. Daneben bedarf es aber auch einer offenen diskursiven Verständigung zwischen den Beratenden und den Beratungsanbietern, inwiefern institutionelle Interessen mit den Beratungen verfolgt werden und wie diese vor dem Hintergrund des professionellen Selbstverständnisses der Beratenden und den Beratungsanliegen der Ratsuchenden bearbeitet werden. Dies bedeutet auch, dass Beratende nicht nur reflektieren sollten, welche Einflüsse die Beratungsanbieter nehmen, sondern auch die der jeweiligen Träger. Die Bildungspolitik ist m. E. gehalten, umsichtiger zu planen, bei welchen Trägern die kommunalen Weiterbildungsberatungsstellen formal und lokal angesiedelt werden. Eine Möglichkeit wäre eine Verortung in Lernzentren, die dem Modell des „One-Stop-Shop“ (Stang 2011, S. 9) folgen, in denen unterschiedliche Beratungsformate (Weiterbildungsberatung, Kompetenzentwicklungsberatungen, Förderberatungen und Lernberatungen) bereits jetzt einen großen Stellenwert haben. Dort könnten einrichtungsgebundene mit einrichtungsübergreifenden Beratungen lokal verzahnt werden (vgl. ebd., S. 16 f.).

Open Access Dieser Artikel wird unter der Creative Commons Namensnennung 4.0 International Lizenz (http://creativecommons.org/licenses/by/4.0/deed.de) veröffentlicht, welche die uneingeschränkte Nutzung, Verbreitung und Wiedergabe für beliebige Zwecke erlaubt, sofern Sie den/die ursprünglichen Autor(en) und die Quelle ordnungsgemäß nennen, einen Link zur Creative Commons Lizenz beifügen und angeben, ob Änderungen vorgenommen wurden.

\section{Literatur}

Becker-Mrotzek, M. (2001). Gespräche in Ämtern und Behörden. In K. Brinker, G. Antos, W. Heinemann \& S. V. Sager (Hrsg.), Text- und Gesprächslinguistik. Linguistics of Text and Conversation. Ein internationales Handbuch zeitgenössischer Forschung. An International Handbook of Contemporary Research (2. Halbband, S. 1505-1525). Berlin: De Gruyter. 
Deppermann, A. (2000). Ethnographische Gesprächsanalyse. Zu Nutzen und Notwendigkeit von Ethnographie für die Konversationsanalyse. Gesprächsforschung - Online-Zeitschrift zur verbalen Interaktion (1). www.gespraechsforschung-ozs.de/heft2000/ga-deppermann.pdf. Zugegriffen: 13. Dez. 2014.

Deppermann, A. (2010). Zur Einführung: „Verstehen in professionellen Handlungsfeldern“ als Gegenstand einer ethnographischen Konversationsanalyse. In A. Deppermann, U. Reitemeier, R. Schmitt, \& T. Spranz-Fogasy (Hrsg.), Verstehen in professionellen Handlungsfeldern (S. 7-25). Tübingen: Gunter Narr Verlag.

Deutscher Städtetag (o. J.). Kommunale Bildungs- und Weiterbildungsberatung. Köln. www.staedtetag.de/ imperia/md/content/dst/reader_weiterbildung.pdf. Zugegriffen: 13. Dez. 2014.

Disse, F. (2005). „Das habe ich nicht gesagt das sagen Sie“. Interaktionen und ihre Bedeutung für den Beratungsprozess am Beispiel von Arbeitsberatungen. Eine Interaktionsanalytische Interpretation (unveröffentlichte Diplomarbeit). Dortmund.

Drew, P., \& Heritage, J. (1992). Analyzing talk at work: An introduction. In P. Drew \& J. Heritage (Hrsg.), Talk at work: Interaction in institutional settings (S. 3-65). Cambridge: Cambridge University Press.

Eberle, T. S. (1997). Ethnomethodologische Konversationsanalyse. In R. Hitzler \& A. Honer (Hrsg.), Sozialwissenschaftliche Hermeneutik (S. 245-279). Opladen: UTB. für Wissenschaft.

Faulstich, P., \& Zeuner, C. (2010). Erwachsenenbildung. Weinheim: Belz

Haas, M., Schiersmann, C., \& Weber, P. C. (2012). Ausführliche Darstellung des Kompetenzprofils für Beratend. In. Nationales Forum Beratung in Bildung, Beruf und Beschäftigung (nfb) und Forschungsgruppe Beratungsqualität am Institut für Bildungswissenschaft der Ruprecht-Karls-Universität Heidelberg (Hrsg.), Kompetenzprofil für Beratende. Ergebnis aus dem Verbundprojekt: Offener Koordinierungsprozess Qualitätsentwicklung in der Beratung für Bildung, Beruf und Beschäftigung. www.elgpn.eu/elgpndb/fileserver/files/51. Zugegriffen: 13. Dez. 2014.

Käpplinger, B. (2010). Nutzen von Bildungsberatung. DIE Zeitschrift, 18(2), 32-35.

Käpplinger, B., \& Klein, R. (2013). Beratung bei Weiterbildungsgutscheinen: Zwischen Prüfung, Information und Entscheidungshilfe. In B. Käpplinger, R. Klein, \& E. Haberzeth (Hrsg.), Weiterbildungsgutscheine. Wirkung eines Finanzierungsmodells in vier europäischen Ländern (S. 15-38). Bielefeld: W. Bertelsmann.

Mader, W. (1999). Weiterbildung und Beratung. In R. Tippelt (Hrsg.), Handbuch Erwachsenen-/Weiterbildung (S. 318-326). Opladen: Leske und Budrich.

Maier-Gutheil, C. (2009). Zwischen Beratung und Begutachtung. Pädagogische Professionalität in der Existenzgründungsberatung. Wiesbaden: VS Verlag für Sozialwissenschaften.

Mcleod, J., \& Machin, L. (1998). The context of counseling. A neglected dimension of trainings research and practice. British Journal of Guidance and Counselling, 26, 325-336.

Möller, S. (2011). Marketing in der Erwachsenenbildung. Bielefeld: W. Bertelsmann.

Müller, A. (2005). Weiterbildungsberatung. Qualitative Analyse von Interaktions- und Prozessverläufen situativer und biographieorientierter Weiterbildungsberatungsgespräche. Berlin: Rhombos-Verlag.

Nolda, S. (2000). Interaktion in pädagogischen Institutionen. Opladen: Barbara Budrich.

Pfadenhauer, M., \& Brosziewski, A. (2008). Professionelle in Organisationen - Lehrkräfte in der Schule. Eine wissenssoziologische Perspektive. In W. Helsper, S. Busse, M. Hummrich, \& R.-T. Kramer (Hrsg.), Pädagogische Professionalität in Organisationen. Neue Verhältnisbestimmungen am Beispiel der Schule (S. 79-97). Wiesbaden: VS Verlag für Sozialwissenschaften.

Pörtner, S. (2006). Anforderungsstruktur und Praktiken der Berufswahlberatung. Eine interaktionsanalytische Untersuchung von Berufsberatungsgesprächen. http://publikationen.ub.uni-frankfurt.de/ files/667/Dissertation_Anforderungsstruktur_und_Praktiken_der_Berufswahlberatung.pdf. Zugegriffen: 13. Dez. $201 \overline{4}$.

Schiersmann, C. (2009). Beratung im Kontext des lebenslangen Lernens. In R. Tippelt \& A. von Hippel (Hrsg.), Handbuch Erwachsenenbildung/Weiterbildung (S. 747-767). Wiesbaden: VS Verlag für Sozialwissenschaften.

Schiersmann, C. (2011). Beratung im Kontext von Lebenslangem Lernen und Life Design - ein Generationen übergreifendes Konzept. In T. Eckert, A. von Hippel, M. Pietraß, \& B. Schmidt-Hertha (Hrsg.), Bildung der Generationen (S. 427-439). Wiesbaden: VS Verlag für Sozialwissenschaften.

Schiersmann, C., \& Remmele, H. (2004). Beratungsfelder in der Weiterbildung. Eine empirische Bestandsaufnahme. Baltmannsweiler: Schneider.

Stang, R. (2011). Strukturen und Leistungen von Lernzentren. Empirische Befunde und Perspektiven zur Entwicklung von kommunalen Lernzentren als innovative Institutionalformen für Lebenslanges Lernen. www.die-bonn.de/doks/2011-lernzentrum-01.pdf. Zugegriffen: 22. Jan. 2015. 
Stanik, T. (2014). „Steht ja im Programm“ - Kurswahlberatung am Beispiel einer Englischeinstufungsberatung. In A. Schlüter (Hrsg.), Beratungsfälle - Fallanalysen für Lern- und Bildungsberatung (S. 129-143). Opladen: Barbara Budrich.

Tippelt, R. (1997). Beratung in der Weiterbildung: Grundlagen und Perspektiven. In T. Eckert, C. Schiersmann, \& R. Tippelt (Hrsg.), Beratung und Informationen in der Weiterbildung (S. 1-69). Baltmannsweiler: Schneider.

Tippelt, R. (2009). Institutionenforschung in der Erwachsenen-/Weiterbildung. In R. Tippelt \& A. von Hippel (Hrsg.), Handbuch Erwachsenen/Weiterbildung (S. 453-471). Wiesbaden: VS Verlag für Sozialwissenschaften. 